\title{
Rendimiento Aeróbico en Niños Obesos de 6 a 10 Años
}

\author{
ANTONIO LÓPEZ S. ${ }^{1,2}$, LORENA SOTOMAYOR S. ${ }^{1,3}$, MARÍA PAZ ÁLVAREZ G. ${ }^{1}$, \\ PAUlA CÉSPEDES A. ${ }^{1}$, CAROLINA POBLETE A. ${ }^{1}$, PAULA VÁSQUEZ M. ${ }^{1}$, MÁXIMO ESCOBAR C. ${ }^{1,2}$ \\ 1. Kinesiólogo. \\ 2. Departamento de Kinesiología, Universidad Católica del Maule. \\ 3. Departamento de Kinesiología, Universidad Metropolitana de Ciencias de la Educación.
}

\begin{abstract}
Aerobic Performance in Obese Children of 6 to 10 Years Old

Objective: To study the relationship between aerobic performance and nutritional state in children of 6-10 years old. Method: Observational and cross-sectional design. A sample of 98 children (78 girls) attending school in the Metropolitan Region was taken. Of these, 47 were diagnosed as obese, 51 were used as controls. Aerobic performance was determined by distance walked (DW) in 6 minutes walk test (6MWT). Continuous measures of reserve heart rate (RHR) and subjective sense of fatigue (SSF) were taken. $t$ or Wilcoxon tests were used for comparison between groups, Pearson coefficient for correlations and multiple regression to evaluate control variables. Significance level was set at $p<0.05$. Results: DW in 6MWT shows differences according to nutritional state $(\mathrm{p}<0.0001)$, with a mean distance of $627.6 \pm 24.5$ $\mathrm{m}$ in control children $v s 560 \pm 26.8 \mathrm{~m}$ in obese children; but these differences are independent of the gender and age variables. Values for RHR and SSF were similar for both groups except for SSF on the first minute. WD and body mass index showed inverse correlation $(r=-0,58 ; p<0.0001)$. Conclusions: The performance in 6MWT was lower among obese children, showing an independent relationship with nutritional state and limited cardiorespiratory efficiency, restricting functional activities in daily life.

(Key words: Obesity, children, aerobic performance).

Rev Chil Pediatr 2009; 80 (5): 444-450
\end{abstract}

\section{RESUMEN}

Objetivo: Estudiar la relación entre rendimiento aeróbico y estado nutricional (EN) en escolares de 6 a 10 años. Método: Diseño observacional, corte transversal. Muestra: 98 escolares (78 mujeres), 51 controles (C) y 47 obesos (OB), de colegios de la Región Metropolitana. El rendimiento aeróbico se evaluó con distancia recorrida (DR) en el test de marcha en 6 minutos (TM6). Se estimó continuamente frecuencia cardíaca de reserva (FCR) y sensación subjetiva de fatiga (SSF). Para comparar medias, se usó test $t$ o Wilcoxon; como índice de correlación, Pearson y para variables de control, regresión lineal múltiple; nivel

Trabajo recibido el 10 de septiembre de 2008, devuelto para corregir el 04 de mayo de 2009, segunda versión el 03 de agosto de 2009, aceptado para publicación el 10 de agosto de 2009.

Fuente Financiamiento: Fondos Proyectos de Investigación DIUMCE, código FIBAS 16-07, 2007.

Correspondencia a:

Antonio López S.

E-mail: alopez@ucm.cl 
de significancia, $\mathrm{p}<0,05$. Resultados: La DR en el TM6 se relacionó con EN ( $\mathrm{p}<0,0001)$, con media de DR $627,6 \pm 24,5$ vs $560 \pm 26,8 \mathrm{~m}$, en niños $\mathrm{C}$ y OB, respectivamente, asociación que no fue modificada por edad y género. Los valores de \%FCR y SSF fueron similares en los grupos, excepto la SSF del minuto 1. DR e IMC mostraron asociación inversa moderada $(r=-0,58 ; p<0,0001)$. Conclusión: El rendimiento en el TM6 fue menor en $\mathrm{OB}$, mostrando una relación independiente con EN y limitada eficiencia cardiorrespiratoria, lo cual puede restringir la capacidad funcional en actividades de la vida diaria.

(Palabras clave: Obesidad, niños, rendimiento aeróbico).

Rev Chil Pediatr 2009; 80 (5): 444-450

\section{Introducción}

La obesidad infantil tiene múltiples consecuencias negativas para la salud, entre las cuales se describen alteraciones a nivel orgánico, psicológico y social ${ }^{1-3}$. El sedentarismo y una limitada capacidad física son factores que contribuyen al desarrollo de obesidad infantil. Al respecto, la aptitud aeróbica es un atributo físico asociado a la condición de salud cardiovascular en niños y adolescentes y, en forma específica, con el exceso de adiposidad corporal ${ }^{4}$. La presencia de factores orgánicos, conductuales y hábitos de vida pueden afectar el óptimo desarrollo de una buena aptitud aeróbica ${ }^{4,5}$.

En tal contexto, el rendimiento aeróbico expresa una capacidad fisiológica que sustenta la ejecución de tareas físicas que demandan al sistema cardiorrespiratorio. A su vez, el rendimiento y la respuesta individual al ejercicio de tolerancia reflejan el estado funcional de los sistemas respiratorio, cardiovascular, neuromuscular y metabólico. Esta información tiene utilidad clínica tanto para objetivos de diagnóstico, monitoreo y evaluación de resultados en salud 6,7 .

Diversos estudios han demostrado que en población infantil, existe una relación entre rendimiento físico en test aeróbicos y estado nutricional ${ }^{8-11}$. Se ha reportado que una limitación de este tipo, aumenta el riesgo de desarrollar obesidad en niños normopeso ${ }^{12}$. El deterioro del rendimiento aeróbico observado en niños con sobrepeso y obesos se atribuye a tres factores: a) el exceso de grasa corporal actúa como carga extra; b) restricciones fisiológicas del sistema cardiorrespiratorio y c) baja motivación para ejecutar actividades de larga duración $^{5,6}$.

En Chile, Kain J. y et a ${ }^{13}$, reportaron una relación entre estado nutricional y capacidad aeróbica medida con test de campo en una muestra de escolares de 8 a 14 años. El estudio permitió establecer que niños de ambos géneros con sobrepeso y obesidad tienen menor capacidad aeróbica. Sin embargo, estos hallazgos son derivados de la aplicación de un test físico con limitada aplicabilidad en contextos clínicos.

La aplicación del TM6 es una buena opción para estimar rendimiento aeróbico en poblaciones pediátricas con enfermedades crónicas ${ }^{14,15}$. El test de marcha en seis minutos (TM6) es una prueba basada en rendimiento, útil para tal propósito, que cuenta con adecuadas propiedades de confiabilidad y validez ${ }^{7,14-16}$. A nivel nacional, no existen reportes acerca del rendimiento aeróbico de niños obesos medidos con TM6. Este tipo de evaluación permite aumentar la comprensión de las consecuencias funcionales del exceso de grasa corporal en niños, específicamente respecto al potencial déficit cardiorrespiratorio en ejercicio. Esta información puede ser útil para generar estrategias preventivas y terapéuticas con relación a esta población de interés.

Los objetivos de este estudio fueron a) evaluar la asociación entre obesidad y rendimiento aeróbico en ejercicio en escolares chilenos de 6 a 10 años y b) analizar el rol de algunas características generales en el rendimiento cardiorrespiratorio en ejercicio en escolares chilenos obesos.

\section{Sujetos y Método}

Diseño: Observacional, analítico, de corte transversal.

Sujetos: La muestra fue por conveniencia basada en escolares pertenecientes a dos cole- 
gios municipalizados de las comunas de Macul y Ñuñoa, Región Metropolitana, Chile; establecimientos de nivel socioeconómico bajo y medio-bajo. Para incluir los niños al estudio los padres o responsables directos firmaron un Consentimiento Informado. Se seleccionaron escolares de 6 a 10 años, de ambos géneros, que conformaron dos grupos: Controles $(\mathrm{C})$ y obesos (OB). Se excluyeron niños con restricciones médicas para realizar actividad física o presencia de alguna condición limitante para la marcha. Los niños incluidos en este reporte fueron objeto de mediciones independientes que no afectan el rendimiento en el TM6.

Mediciones: Cuatro evaluadores entrenados realizaron la recolección de datos en las dependencias de los colegios.

Evaluación antropométrica: Para la medición de peso y talla se utilizó una balanza con estadiómetro. El niño se evaluó descalzo y con el mínimo de ropa, en posición bípeda, brazos colgando y pies levemente separados. Se calculó IMC en $\mathrm{kg} / \mathrm{m}^{2}$ y según valores normativos en percentiles se clasificó al niño como peso normal (p5-p85) u OB (> p95) de acuerdo a los estándares descritos por Barrera ${ }^{17}$.

Rendimiento aeróbico: Se aplicó el TM6 de acuerdo a los procedimientos descritos por Escobar y $\operatorname{cols}^{18}$. En una pista plana de $30 \mathrm{~m}$ demarcada cada $5 \mathrm{~m}$, se solicitó al niño caminar lo más rápido posible sin correr; antes del test, se realizó explicación y demostración para asegurar comprensión. Además de distancia recorrida (DR), se registró continuamente frecuencia cardíaca (FC) y sensación subjetiva de fatiga (SSF) durante el test y por tres minutos postejercicio. Para obtener el máximo rendimiento, el niño fue estimulado frecuentemente durante la ejecución del test. Un evaluador acompañó al niño durante la caminata con el fin de obtener los datos de FC y SSF.

Respuesta de la FC de reserva y SSF al esfuerzo: Con un telémetro de pulso (Polar $\AA$ ) ubicado en el tórax del niño, se registró la FC en reposo, durante y posterior al test. A partir de estos datos, se calculó la FC de reserva (FCR) según la fórmula de Karvonen la cual se expresó en porcentaje utilizado. Esta variable fue utilizada como indicador de los ajustes cardiovasculares al esfuerzo ${ }^{19}$. La SSF se estimó mediante la escala de Borg modificada (rango 0 a 10) la cual permite relacionar la intensidad de ejercicio con la percepción del esfuerzo y que ha sido validada para ser usada en niños ${ }^{20}$.

Tamaño de muestra y potencia estadística: Según datos de rendimiento en TM6 en niños de peso normal y OB previamente publi$\operatorname{cados}^{18,21}$, se estimó la diferencia intergrupos esperada y se calculó un tamaño de efecto estandarizado de 0,9; así, para obtener una potencia estadística de $80 \%$, con un nivel alfa de 0,05 , fue necesario conformar grupos de al menos 36 niños ${ }^{22}$.

Análisis de datos: Para estadística descriptiva se usó media y desviación estándar (mediana cuando se indica). Para comparación de medias se aplicó el test $t$; en datos con distribución asimétrica, la prueba de suma de rangos de Wilcoxon. La correlación se estimó con el coeficiente de Pearson. El nivel de significancia se fijó con un $\mathrm{p}<0,05$. Para evaluar la influencia de género y edad en el rendimiento en el TM, se usó Regresión Lineal Múltiple. En todos los procedimientos se usó el programa estadístico Stata $9.0^{\circledR}$.

\section{Resultados}

Se evaluaron 98 niños de ambos géneros; todos completaron el protocolo sin inconvenientes. Las características demográficas y antropométricas de la muestra se muestran en la tabla 1. La media de edad es similar en ambos grupos, no existiendo diferencias estadísticamente significativas $(\mathrm{p}=0,876)$. La muestra tuvo un predominio del género femenino.

Tabla 1. Características de la muestra según estado nutricional

\begin{tabular}{lll}
\hline & Controles & Obesos \\
\hline Edad, años & $7,8(1,3)$ & $7,8(0,9)$ \\
IMC, kg/m² & $17,0(1,1)$ & $22,1(2,2)$ \\
Género, F/M & $42 / 9$ & $39 / 8$ \\
Colegio $^{+}$1/2 & $12 / 39$ & $14 / 33$ \\
\hline
\end{tabular}

+ Colegios 1: Mercedes Marín del Solar (Macul); 2: Preciosa Sangre (Ñuñoa). Datos presentados con medias (desviación estándar) y frecuencias. 


\section{Rendimiento en TM6 según estado nutricional}

Los resultados en el TM6 según estado nutricional se muestran en la figura 1. La media de DR fue de $627,6 \mathrm{~m}$ (DE: 24,5) y de 560,3 m (DE: 26,8), en niños C y OB, respectivamente. La aplicación de la prueba $t$ confirmó que

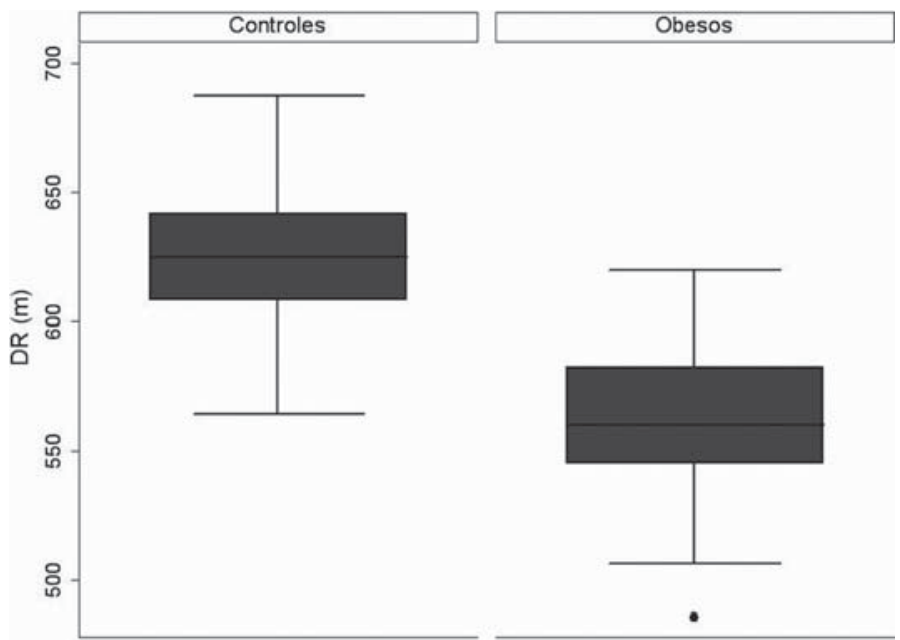

Figura 1. Distancia recorrida (DR) en el TM6 según estado nutricional.

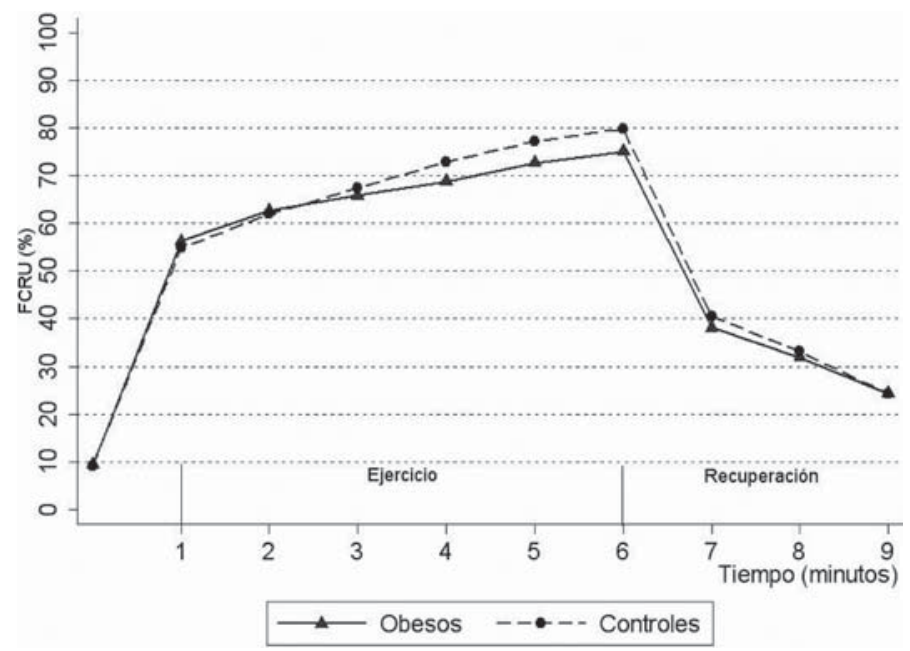

Figura 2. Cambios en la frecuencia cardíaca de reserva utilizada (FCRU) durante el TM6 en niños controles y obesos. las diferencias son significativas $(\mathrm{p}$ $<0,0001)$. Con relación a DR y edad, para el rango de 6 a 10 años, se observó que en el grupo de peso normal el rendimiento aumentó en $63,4 \mathrm{~m}$ y en OB sólo en 31,4 m.

El análisis de regresión múltiple mostró que la relación de DR y estado nutricional es independiente de las características demográficas de los niños. Edad y género no son modificadoras de efecto $(p=0,48 y$ $\mathrm{p}=0,52$, respectivamente) y tampoco afectan el coeficiente de regresión de estado nutricional (-67,26; IC95\%: -74, 94-58, 49; $\mathrm{p}<0,001$ ), es decir, no actúan como factores de confusión. Se determinó que estado nutricional por sí solo explica el 63,3\% de la variabilidad del rendimiento en el TM6. En definitiva, con un nivel de confianza del $95 \%$, se puede afirmar que los niños obesos en promedio alcanzaron 67,26 metros menos que sus pares no obesos.

\section{Respuesta de la FCR y SSF en el TM6}

El registro minuto a minuto de la FCR y la SSF durante el TM6 permite observar el comportamiento de estas variables según se muestra en la figura 2 y tabla 2 , respectivamente. Al respecto, en ambos grupos se observa un comportamiento que refleja la respuesta cardiovascular ante un ejercicio submáximo; es decir, al inicio del ejercicio existe un incremento importante del uso del \%FCR (55,5\%). Esta mag-

Tabla 2. SSF durante el TM6 en tiempos de ejercicio y recuperación en niños de 6 a 10 años

\begin{tabular}{lcccccccccc} 
& Reposo & \multicolumn{4}{c}{} & \multicolumn{2}{c}{ Ejercicio } & \multicolumn{3}{c}{ Recuperación } \\
\hline Controles & 0 & 0 & 2 & 4 & 5 & 5 & 6 & 5 & 0 & 0 \\
Obesos & 0 & 1 & 3 & 5 & 5 & 6 & 7 & 5 & 1,5 & 0 \\
\hline
\end{tabular}

Abreviaciones: $\mathrm{SSF}=$ sensación subjetiva de fatiga. Valores expresados en medianas. 
nitud se acentúa progresivamente hasta el minuto 6 en el cual se obtiene el valor máximo (77,5\%). En el tiempo de recuperación ocurre el fenómeno inverso, existiendo una caída progresiva de los valores hasta el último minuto de recuperación $(24,3 \%)$. Se observa, además, que aparentemente no hay diferencias significativas en los valores de $\%$ FCR según estado nutricional.

Para efectos de comparación intergrupo se analizaron los minutos 1 y 6 de ejercicio y 3 de recuperación dada su significancia fisiológica. De esta forma, por medio del tests $t$ se estableció que no existen diferencias significativas en el uso del \%FCR en el minuto 1 de ejercicio y 3 de recuperación ( $\mathrm{p}=0,7254$ y 0,5099, respectivamente); lo mismo ocurrió en el minuto 6 de ejercicio al aplicar Wilcoxon $(\mathrm{p}=0,1160)$.

Correlación entre rendimiento en TM6 y el IMC: Existe correlación entre la DR y el valor de IMC; a medida que aumenta el IMC, disminuye el rendimiento aeróbico, lo que se expresa en una relación inversa con valor $\mathrm{r}$ de Pearson de $-0,58(p<0,0001)$.

\section{Discusión}

A nivel nacional este es el primer reporte que estudia la relación entre rendimiento aeróbico y estado nutricional en escolares por medio de la aplicación del TM6. Se estableció una relación entre el rendimiento alcanzado en el TM6 y el estado nutricional de escolares de 6 a 10 años. Además, por medio de análisis multivariable, se determinó que dicha relación es independiente de las características demográficas edad y género. Luego, en comparación con sus pares de peso normal, los niños obesos presentaron una disminución promedio de 67,26 m en la prueba de caminata.

La limitación funcional cardiorrespiratoria es un hallazgo consistente en muestras de niños $\mathrm{y}$ adolescentes obesos ${ }^{8-13}$. Day et $\mathrm{al}^{21}$, compararon el rendimiento en el TM6 en 30 escolares obesos con 30 no obesos de 8 a 10 años; media (DE) en DR de 664,4 (31,0) $\mathrm{m}$ y 702,1 $(33,3) \mathrm{m}$, respectivamente. Por su parte, Drinkard $\mathrm{B}^{11}$, estableció en una muestra de adolescentes obesos una correlación entre composición corpo- ral, capacidad aeróbica medida con un test ergométrico y rendimiento en una prueba de caminata de 12 minutos; de acuerdo al registro de la frecuencia cardíaca antes-después de la prueba, se observó una menor reserva cardiovascular, sin embargo, la media de IMC de la muestra $\left(42,9 \mathrm{~kg} / \mathrm{m}^{2}\right)$ es muy superior respecto a nuestro estudio $\left(22,1 \mathrm{~kg} / \mathrm{m}^{2}\right)$. Graf et $\mathrm{al}^{10}$, estudiaron 513 escolares de 5 a 14 años, demostrando una relación entre estado nutricional y tolerancia al ejercicio medido con un test de carrera de 6 minutos. En concordancia con nuestros resultados, destacan que dicha asociación se mantiene luego de ajustar el análisis por género y edad. Asimismo, con un diseño basado en la comunidad, Eisenmann et al $^{9}$ detectaron en adolescentes una asociación independiente entre IMC y la capacidad aeróbica, relacionando lo anterior con la presencia de factores de riesgo cardiovascular; a mayor IMC y menor aptitud aeróbica, peor perfil de factores de riesgo.

Nuestros resultados confirman que la condición de obeso predispone a un déficit en la eficiencia del sistema cardiorrespiratorio durante demandas físicas de tipo aeróbico. $\mathrm{La}$ magnitud de esta limitación puede estar asociada al grado de aumento del IMC ${ }^{8,11,13}$; en este estudio, se observó una asociación inversa moderada entre DR e IMC.

En el contexto de la salud física del niño, un déficit funcional de esta naturaleza puede tener las siguientes consecuencias: a) menor rendimiento en esfuerzos físicos de tolerancia, limitando la capacidad en actividades de la vida diaria; b) potencial asociación del deterioro cardiorrespiratorio con la aparición de factores de riesgo cardiovascular; c) capacidad reducida para sostener actividades de tolerancia necesarias para aumentar el consumo energético y así reducir la grasa corporal.

Al combinar el análisis de rendimiento y costo fisiológico durante la aplicación del TM6, los niños obesos presentaron niveles similares de uso porcentual de la FCR pero con rendimiento inferior; esto puede indicar menor eficiencia cardiorrespiratoria. Tales hallazgos son similares a lo reportado por Norman et $\mathrm{al}^{8}$, quienes al aplicar test de 12 minutos en base a caminar y/o correr en niños con obesidad, ob- 
servaron menor DR y valores máximos de FC inferiores respecto a sus pares no obesos. Sin embargo, la muestra estudiada difiere de la nuestra en dos aspectos significativos: un promedio de edad superior y que los participantes presentaban obesidad mórbida, lo cual puede exacerbar las limitaciones físicas.

Con relación a los cambios en la SSF durante y posterior al ejercicio, observamos una respuesta típica para esta variable; así por ejemplo, el valor máximo se presentó en el minuto 6 (valor 7 de 10). El análisis comparativo mostró que sólo en el minuto 1 hubo diferencias estadísticamente significativas, siendo mayor en los obesos, es decir, solamente al inicio del ejercicio se expresó una mayor percepción del esfuerzo en el grupo OB. Dicho hallazgo está fuera de lo esperado, ya que a medida que transcurre el test, aumenta la carga aeróbica lo cual tiende a incrementar la percepción de esfuerzo hacia el final del test. Al respecto, además del error de medición, es posible que la escala de Borg cuente con limitada capacidad discriminativa.

En esta investigación se usó un protocolo de test funcional para evaluar el rendimiento aeróbico. El TM6 está validado para ser usado en niños sanos y en grupos pediátricos con distintas condiciones de salud ${ }^{14-16}$. Esta prueba de ejercicio se ha convertido en una herramienta de medición clínica de gran utilidad debido a que cuenta con adecuada capacidad discriminativa, predictiva y para evaluar cambios producto de intervenciones ${ }^{7,15,23,24}$. En nuestro estudio, junto con los valores de DR se monitorizó el costo fisiológico en ejercicio y fase de recuperación como método de estimación de la eficiencia aeróbica, cualidad que se observó restringida en niños $\mathrm{OB}$.

Una posible limitación de esta investigación es el predominio del género femenino en la muestra. En teoría, esto podría afectar el rendimiento promedio en DR para ambos grupos, sin embargo, esto no impidió demostrar deterioro en la tolerancia aeróbica del grupo OB. Por otra parte, el análisis multivariable confirmó una asociación independiente entre rendimiento en el TM6 y estado nutricional.

La población de estudio permite extrapolar los resultados a niños obesos del rango de edad especificado y que pertenezcan a colegios de perfil socio-geográfico similar al de esta investigación. En nuestra opinión, es inconveniente la aplicación de los hallazgos a muestras clínicas de niños obesos o escolares con grado severo de obesidad, debido que en éstas se pueden presentar co-morbilidades y otros factores orgánicos distintos a los de nuestra población de estudio.

En síntesis, el rendimiento aeróbico estimado con el TM6 está reducido en escolares obesos de 6 a 10 años. En tal sentido, la capacidad funcional presentó relación con el estado nutricional, independiente de las variables demográficas edad y género. El comportamiento de la respuesta fisiológica reveló menor eficiencia cardiorrespiratoria en niños obesos. Estos resultados confirman que la obesidad infantil tiene como consecuencia un deterioro funcional cardiorrespiratorio expresado en esfuerzos de tolerancia, lo cual genera desventajas en el desempeño físico aeróbico y predispone a consecuencias negativas para la salud física del niño.

\section{Referencias}

1.- Burrows R: Obesidad infantil y juvenil: consecuencias sobre la salud y calidad de vida futura. Rev Chil Nutr 2000; 27 (1): 141-8.

2.- World Health Organization: Obesity: preventing and managing the global epidemic. Report of a WHO Consultation on Obesity, Ginebra, 3-5 Junio, 1997.

3.- Hills AP, Hennig EM, Byrne NM, Steele JR: The biomechanics of adiposity-structural and functional limitations of obesity and implications for movement. Obes Rev 2002; 3: 35-43.

4.- Ortega FB, Ruiz JR, Castillo MJ, Sjöström M: Physical fitness in childhood and adolescence: a powerful marker of health. Int J Obes 2008; 32: 1-11.

5.- Rowland $T W$ : Maturation of aerobic fitness. En: Developmental exercise physiology. USA: Human Kinetics, 1996. Capítulo 6: 73-97.

6.- López S, Escobar M: Pruebas funcionales en niños. Revista Kinesiología 2004; 73: 115-9.

7.- Solway S, Brooks D, Lacasse Y, Thomas S: A qualitative systematic overview of the measurement properties of functional walk tests used in the cardiorespiratory domain. Chest 2001; 119: 256-70. 
8.- Norman A-C, Drinkard B, McDuffie J, Ghorbani S, Yanoff L, Yanovski J: Influence of excess adiposity on exercise fitness and performance in overweight children and adolescents. Pediatrics 2005; 115: 6906.

9.- Eisenmann JC, Katzmarzyk PT, Perusse L, Tremblay A, Deprés J-P, $M$ Bouchard C: Aerobic fitness, body mass index and CVD risk factors among adolescents: the Québec family study. Int J Obes 2005; 29: 1077 83.

10.- Graf G, Koch B, Kretschmann-Kandel E, et al: Correlation between BMI, leisure habits and motor abilities in childhood. (Child-Proyect). Int J Obes 2003; 28: 22-6.

11.- Drinkard B, McDuffie J, McCann S, Uwaifo G, Nicholson J, Yanovski: Relationships between walk/ run performance and cardiorespiratory fitness in adolescents who are overweight. Phys Ther 2001; 81: 1889-96.

12.- Kim J, Must A, Fitzmaurice G, et al: Relationship of physical fitness to prevalence and incidence of overweight among schoolchildren. Obes Res 2005; 13: 1246-54.

13.- Kain J, Olivares $S$, Romo $M$, et al: Estado nutricional y resistencia aeróbica en escolares de educación básica: línea de base de un Proyecto de Promoción de Salud. Rev Méd Chile 2004; 132: 1395-402.

14.- Noonan V, Dean E: Submaximal exercise testing: clinical application and interpretation. Phys Ther 2000; 80: 782-807.

15.- Nixon PA, Joswiak M, Fricker FJ: A six-minute walk test for assessing exercise tolerance in severely children. J Pediatr 1996; 129: 362-6.

16.- Finch E, et al: Physical rehabilitation outcome measures. A guide to enhanced clinical decision making. $2^{\circ}$ ed. Ontario, Canada: Lippincott, Williams \& Wilkins, 2002.

17.- Barrera GM: Estándares antropométricos para evaluación del estado nutritivo. Santiago: Instituto de $\mathrm{Nu}-$ trición y Tecnología de Alimentos (INTA) Universidad de Chile, 2004.

18.- Escobar M, López S, Véliz C, Crisóstomo S, Pinochet $R$ : Test de marcha en 6 minutos en niños chilenos sanos. Revista Kinesiología 2001; 62: 16-20.

19.- Heyward $V$ : Advanced fitness assessment exercise prescription. $3^{\text {a }}$ ed. Philadelphia, USA: Human Kinestics, 1998.

20.- Eakin B, Finta K, Serwer G, Beekman R: Perceived exertion and exercise intensity in children with or without structural heart defects. J Pediatr 1992; 120 : 90-3.

21.- Day A, Misto L, Rojas P: Rendimiento funcional y respuesta de la presión arterial postejercicio medido en test de marcha en seis minutos en niños escolares obesos. Tesis para optar al título de Kinesiólogo. UMCE, Carrera de Kinesiología, 2004.

22.- Browner $W$, Newman T, Cummings $S$, Hulley $S$ : Estimating sample size and power: the nitty-gritty. En: Designing clinical research. $2^{\circ}$ ed. Philadelphia, USA: Ed. Lippincott Williams \& Wilkins, 2001; Capítulo 6:65-91.

23.- Moalla W, Gauthier R, Maingourd W, Ahmaidi S: Six-minute walking test to assess exercise tolerance and cardiorespiratory responses during training program in children with congenital heart disease. Int J Sports Med 2005; 26: 756-62.

24.- Calders $P$, Deforche B, Verschelde $S$, et al: Predictors of 6-minute walk test and 12-minute walk/run test in obese children and adolescents. Eur J Ped 2008; 167 : 563-8. 